memo (2010) Vol. 3: 47-48

DOI 10.1007/s12254-010-0186-7

Printed in Austria

(C) Springer-Verlag 2010

\title{
Current treatment of gastrointestinal stromal tumours
}

\author{
V. Tzekova \\ University Hospital "QueenJoanna”, Sofia, Bulgaria
}

Gastrointestinal stromal tumours (GIST) are the most common mesenchymal tumours of the gastrointestinal tract. The seminal discovery that these tumours express the KIT tyrosine kinase and commonly harbour oncogenic mutations in the KIT gene provided the rationale for targeted therapy with tyrosine kinase inhibitors. The implementation of imatinib mesylate (Glivec) revolutionized the treatment of GIST as this drug achieved three times longer overall survival comparing with the historical controls in the advanced disease.

M. Kamenova [1] reviewed comprehensively the data on pathomorphological and genetic characteristics of GIST in her contribution "Pathology of Gastrointestinal Stromal Tumours with Accent to Prognostic Criteria". The author described in details the pathomorphological and immunohistochemical features associated with the diagnosis of GIST. She pointed out the role of a positive staining of CD117 as it was found in more than $80 \%$ of these tumours. KIT (CD117) negative tumours usually expressed PDGFR-A mutation. Further, M. Kamenova showed the criteria for GIST classification in regards to the risk of aggressive behaviour - the tumour size and site, and mitotic activity. The author discussed comprehensively the status of different KIT- and PDGFR-A mutations and their role to the prognosis and prediction to target therapy.

The development of a highly specific and effective targeted therapy with imatinib mesylate represents a paradigm shift in the therapy of advanced GIST. G. Kurteva and P. Kurtev [2] reviewed the clinical data in this topic in their paper "Imatinib Mesylate (Glivec) in the Management of Gastrointestinal Stromal Tumours (GIST)". They showed the results of the pivotal clinical study B222 after patients have been treated with imatinib for up to 71 months (median 63 months). Median overall survival (OS) was 58 months, which was a revolutionary step in the management of advanced GIST. Further, the authors showed the results of two phase III clinical studies, EORTC 62005 and S0033, aimed to assess the relationship of the mutational status of KIT- and PDGFR-A to the efficacy of imatinib. The combined analysis of these two studies demonstrated that initiating the imatinib therapy at $800 \mathrm{mg}$ daily dose in patients with KIT exon 9 mutations improved progression-free survival (PFS). These results provide a rationale to individualize the imatinib dosing - $400 \mathrm{mg}$ daily dose in patients with exon 11 mutations and $800 \mathrm{mg}$ daily dose in patients with exon 9 mutations. Another issue addressed in a

Correspondence: Valentina Tzekova, University Hospital

"QueenJoanna", 8 Bjalo more str, 1527 Sofia, Bulgaria.

E-mail: tzekova@techno-link.com phase III clinical study BRF14 was the duration of imatinib therapy in patients with advanced GIST. The results of this trial demonstrated that imatinib should not be discontinued irrespectively of the achieved objective response and durability of the response. The interruption of imatinib in patients who had achieved clinical benefit resulted in a rapid progression of the disease. Kurteva and Kurtev discussed also the safety profile of imatinib. They pointed out that imatinib therapy was discontinued because of drug-related adverse reactions only in $4 \%$ of patients. The most common adverse reactions were vomiting, diarrhoea, abdominal pain, fluid retention and others. In general, imatinib was well tolerated along the treatment for many years. At the end of their manuscript the authors presented the International Guidelines for GIST management.

The treatment of patients with imatinib resistant GIST is a big challenge. C. Timcheva reviewed the data of investigations on primary and secondary imatinib resistance in her paper "Second-Line Targeted Therapy of Gastrointestinal Stromal Tumours" [3]. She pointed out that $5 \%$ of patients experienced primary resistance to imatinib and another $14 \%$ developed early resistance. The mechanisms of primary resistance included decreased absorption of imatinib or binding of the imatinib to the blood components. Secondary resistance developed after a median of about 2 years of treatment with imatinib. It would be a result of different molecular mechanisms among which the acquisition of a new, secondary mutation in KIT or PDGFR-A was the most frequent (up to $80 \%$ ). The resistance to imatinib dosed at $400 \mathrm{mg}$ daily might be overcome by increasing the daily dose to $800 \mathrm{mg}$. Recently, a pharmacokinetic study of imatinib explored the relationship between imatinib trough plasma levels and long-term clinical outcome. The results of this study showed that patients with imatinib trough levels $<1100 \mathrm{ng} / \mathrm{ml}$ experienced a shorter time to progression (TTP) and lower rate of clinical benefit. That is why the monitoring imatinib trough plasma level may be advisable in patients with lack of expected clinical benefit, or compliance concerns. Further, C. Timcheva reviewed the results of clinical studies on second- and thirdline treatment of advanced GIST. The standard treatment after failure of imatinib therapy became sunitinib (a small molecule tyrosine kinase inhibitor) based on the results of phase III study comparing sunitinib with placebo. The benefit of sunitinib therapy was demonstrated in terms of TTP, progression-free survival (PFS) and objective response rate (ORR). Patients who are resistant or intolerable to both imatinib and sunitinib therapy have a poor prognosis and a few 
therapeutic options. A variety of tyrosine kinase inhibitors, such as dasatinib, nilotinib, pazopanib, sorafenib and others, are now under investigation as third-line treatment in advanced GIST.

The risk of recurrence is high after surgery. About 40 $70 \%$ of patients who underwent surgery for primary tumour develop metastases. K. Koynov reviewed in his contribution "Adjuvant Therapy with Imatinib in GIST Patients after Radical Surgery" [4] the results from clinical studies in this topic. He pointed out the significance of patient categorization into four categories according to the risk of recurrence based on tumour size and mitotic count per 50 high power fields. The results of phase II clinical study Z9000 in patients with large tumours (median tumour size of $13 \mathrm{~cm}$ ) showed that imatinib at a dose of $400 \mathrm{mg}$ daily for 1 year following radical surgery improved recurrence-free survival (RFS) and OS in comparison to historical controls. The following phase III study Z9001 confirmed the benefit of adjuvant imatinib for 1 year, especially in patients with bigger tumours. One-year RFS was $97 \%$ for imatinib group and $83 \%$ for placebo group ( $p=0,0000014)$.
Two other clinical trials (EORTC62024 and SS-GXVIII/AIO) are still ongoing. Both of them are addressed to the open question of the duration of adjuvant imatinib therapy. Many other questions concerning the adjuvant therapy in operable GIST are still unanswered. New clinical studies are warranted in order to define ways for individualizing therapy in the adjuvant setting.

\section{Conflict of interest}

The author declares that there is no conflict of interest.

\section{References}

[1] Kamenova M. Pathology of gastrointestinal stromal tumours with accent to prognostic criteria. memo, 3(2): 53-57, 2010

[2] Kurteva GP, Kurtev PF. Imatinib mesylate in the management of gastrointestinal stromal tumours (GIST). memo, 3(2): 49-52, 2010.

[3] Timcheva C. Second-line targeted therapy of gastrointestinal stromal tumours. memo, 3(2): 61-64, 2010.

[4] Koynov KD. Adjuvant therapy with imatinib in GIST patients after radical surgery. memo, 3(2): 58-60, 2010. 